\title{
Cordycepin induces autophagy-mediated C-FLIP degradation and leads to apoptosis in human non-small cell lung cancer cells
}

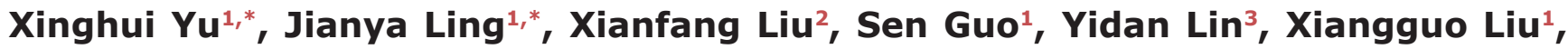 \\ Ling Su ${ }^{1}$ \\ ${ }^{1}$ Shandong Provincial Key Laboratory of Animal Cells and Developmental Biology, Shandong University School of Life \\ Sciences, Jinan, China \\ ${ }^{2}$ The Department of Otolaryngology Head and Neck Surgery, Shandong Provincial Hospital Affiliated to Shandong University, \\ Jinan, China \\ ${ }^{3}$ The Thoracic Surgery Department of West China Hospital, West China Medical School of Sichuan University, Chengdu, China \\ *These authors have contributed equally to this work \\ Correspondence to: Ling Su, email: suling@sdu.edu.cn
}

Keywords: apoptosis, C-FLIP, autophagy, cordycepin

Received: June 16, 2016

Accepted: December 01, 2016

Published: December 27, 2016

\section{ABSTRACT}

Cordycepin, a main active composition extracted from Cordyceps militaris, has been reported to exert anti-tumor activity in a broad spectrum of cancer types. However, the function of cordycepin on human non-small cell lung cancer cells is still obscure. Our present work showed that cordycepin inhibited cell growth by inducing apoptosis and autophagy in human NSCLC cells. Further study revealed that cordycepin triggered extrinsic apoptosis associated with down-regulation of $c-F L I P_{L}$ which suppresses the activity of caspase-8. And ectopic expression of

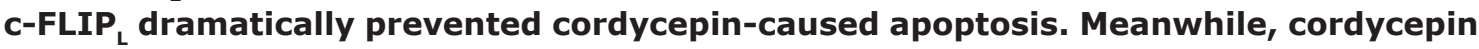
stimulated autophagy through suppressing mTOR signaling pathway in lung cancer cells. When autophagy was blocked by Atg5 siRNA or PI3K inhibitor LY294002, the levels of apoptosis caused by cordycepin were obviously attenuated. In addition, suppression of autophagy could also elevate the level of $C-F L I P_{L}$ which indicated cordycepin-triggered autophagy promoted the degradation of $\mathrm{C}-\mathrm{FLIP}_{\mathrm{L}}$. Therefore, we conclude that cordycepin induces apoptosis through autophagy-mediated downregulation of C-FLIP $P_{L}$ in human NSCLC cells. Taken together, our findings provide a novel prospect on the anti-tumor property of cordycepin, which may further prompt cordycepin to serve as a promising therapeutic approach in NSCLC treatment.

\section{INTRODUCTION}

Lung cancer has been a disastrous malignant neoplasm with highest incidence and mortality all over the world, which represents a poor five-year survival rate of less than $15 \%$ [1]. Hence, it is extraordinarily urgent to develop and exploit novel anticancer agents to improve its clinical outcomes. Nowadays, natural agents have attracted much attention for cancer treatment. Cordycepin (3'-deoxyadenosine), a natural product derived from Cordyceps sinensis, has been widely used in Chinese traditional medicine. Cordycepin possesses multiple pharmacological properties, such as anti-fungal, antibacterial, anti-inflammatory and anti-tumor effects $[2,3]$.
And the anti-cancer ability has been observed in various cancer types including leukemia, gallbladder, colon, prostate, breast, hepatic, oral carcinoma and so on [4]. Cordycepin inhibits cancer cell growth through cell cycle arrest and apoptosis induction [5]. Importantly, Ames and subacute toxicity test showed that cordycepin exhibited non-mutagenic and non-toxic property in rat model by oral administration [6]. However, the effects of cordycepin on human NSCLC cells have not been deeply investigated.

There are two main signaling pathways involved in apoptosis: the extrinsic pathway and the intrinsic pathway. The extrinsic pathway also is named death receptor pathway which is mediated by the stimulation of cell surface receptors when bound to particular ligands. 
Once death receptors are trimerized, death-inducing signaling complex (DISC) is rapidly assembled and leads to the activation of pro-caspase8, thereafter stimulates effector caspases, resulting in apoptosis eventually [7]. Cellular-FLICE inhibitory protein (c-FLIP) is a key antiapoptotic regulator preventing apoptosis via inhibiting caspase 8 activation [8]. The intrinsic pathway, referred to as mitochondrial-mediated pathway, is characterized as increased mitochondrial permeability and release of cytochrome c [9]. Meanwhile, this pathway is tightly regulated by a group of proteins belonging to Bcl-2 family, which consists of pro- and anti-apoptotic proteins. Of note, Bcl-2 and Mcl-1 are well investigated anti-apoptotic members that negatively modulated apoptosis [10].

Autophagy is an evolutionary conserved cellular degradation process by which cytoplasmic components and organelles are packaged into autophagosome, and transported into lysosome for digesting cytoplastic garbage and maintaining cellular homeostasis [11]. This physiological procedure can be activated by diverse cellular stresses, such as nutrient limitation, energy deficiency, oxidative stress, and protein or organelle accumulation [12]. Autophagy possesses dual functions in apoptosis, working as either a suppressor or as a promoter for apoptosis induction [13].

Mammalian target of rapamycin (mTOR) and its modulators are pivotal for cells to sense growth factor, cellular energy and nutrient status, thereby regulates cell growth, protein synthesis and autophagy [12]. The serine/ threonine kinase mTOR is composed of two distinctly different compounds: mTORC1 and mTORC2. mTORC1 is the real sensor to rapamycin and controls protein synthesis by two main substrates: $\mathrm{p} 70$ ribosomal protein S6-kinase (p70S6K) and eukaryotic initiation factor 4E binding protein 1 (EIF4EBP1) [14]. Previous studies demonstrated that inactivation of mTOR contributed to the induction of autophagy by relieving the blockage of Ulk-1 complex [15].

In this study, we found that cordycepin triggered autophagic flux by suppressing mTOR signaling pathway. Additionally, cordycepin-caused autophagy promoted

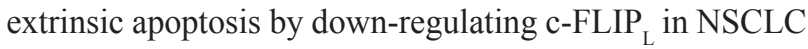
cells. Together, our findings may lay the foundation for cordycepin to develop a novel anticancer agent for tumor treatment.

\section{RESULTS}

\section{Cordycepin induces caspase-dependent apoptosis in human NSCLC cells}

To investigate the effects of cordycepin on cell growth in human lung cancer cells, five NSCLC cell lines (H1792, H1299, H460, H157 and A549) were exposed to increasing concentrations of cordycepin $(0,12.5,25,50$,
$100,200,400 \mu \mathrm{M})$ for $24 \mathrm{~h}$ and $48 \mathrm{~h}$ respectively. Then cell viability was measured by SRB assay. As shown in Figure 1A, the survival of NSCLC cells was significantly reduced in a dose-dependent manner after treatment with cordycepin for $24 \mathrm{~h}$. Then we calculated the halfmaximal inhibitory concentration (IC50) based on the data from SRB assay and found that the IC50 value of H460, H1299 and H157 cell lines was approximately 200 $\mu \mathrm{M}$, while H1792 and A549 had a higher IC50 value of $400 \mu \mathrm{M}$. Moreover, in comparison with control samples, the viability of H460, H1299 and H157 cell lines were suppressed up to $80 \%$ after cordycepin treatment for $48 \mathrm{~h}$, therefore these cell lines were chosen to conduct subsequent analysis.

To illuminate the mechanism that cordycepin caused survival suppression in human NSCLC cells, flow cytometry analysis was performed to examine the impact of cordycepin on apoptosis and the data revealed that cordycepin dramatically increased the percentage of early and late apoptotic cells in H1299 and H460 after treated with increasing dosage of cordycepin for $24 \mathrm{~h}$ (Figure 1B). Afterwards, we carried out western blot assay to detect whether cordycepin triggered caspase-dependent apoptosis and found that the cleaved forms of caspase-8, caspase-9, caspase- 3 and PARP were significantly elevated both in a dose-dependent manner (Figure 1C). Moreover, the time course assay revealed that the cleavage of caspases was appeared at $6 \mathrm{~h}$, and reached the peak at $12 \mathrm{~h}$ (Figure 1D). Therefore, we draw the conclusion that cordycepin leads to caspase-dependent apoptosis in NSCLC.

\section{c-FLIP ${ }_{L}$ is involved in cordycepin-induced apoptosis}

c-FLIP has been shown to be a key negative regulator of extrinsic apoptosis by inhibiting the activation of caspase- 8 in human cancer cells [16]. Thus, we detected whether cordycepin influenced the levels of c-FLIP in NSCLC cells. H1299, H460 and H157 cells were exposed to different concentrations of cordycepin for $12 \mathrm{~h}$ or to 100 $\mu \mathrm{M}$ cordycepin for various times, and the results indicated that c-FLIP ${ }_{\mathrm{L}}$ was greatly down-regulated in a dose and a time-dependent manner (Figure 2A and 2B). Furthermore, we examined whether $\mathrm{c}-F L I P_{\mathrm{L}}$ played a critical role in cordycepin-induced extrinsic apoptosis. H157-FLIP cells $_{\mathrm{L}}$ stably over-expressed c-FLIP ${ }_{L}$ were used to validate the function of c-FLIP $\mathrm{L}_{\mathrm{L}}$ in cordycepin-induced apoptosis. As shown in Figure 2C, the cell viability of H157-FLIP was higher than that in H157-lacz cells especially in the concentration of $200 \mu \mathrm{M}$. What's more, compared with H157-lacz cells, the cleavage of caspase-8, caspase-3 and PARP was significantly weakened in H157-FLIP ${ }_{L}$ cells (Figure 2D). Taken together, downregulation of c-FLIP contributes to cordycepin-induced apoptosis in human lung cancer cells. 
A
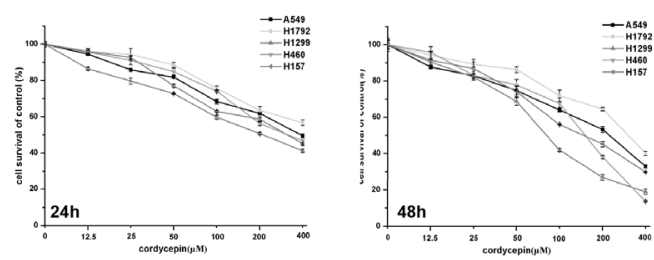

C

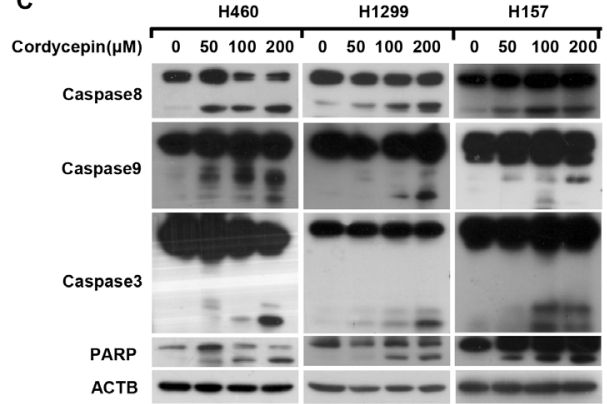

B
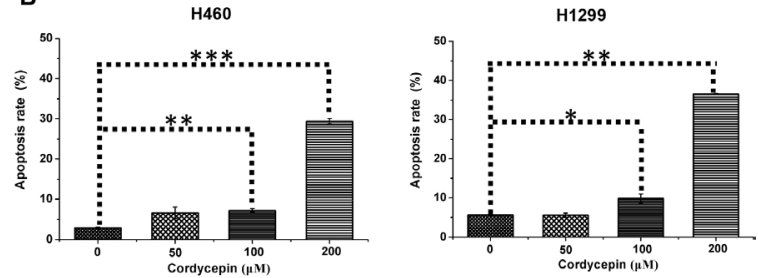

D

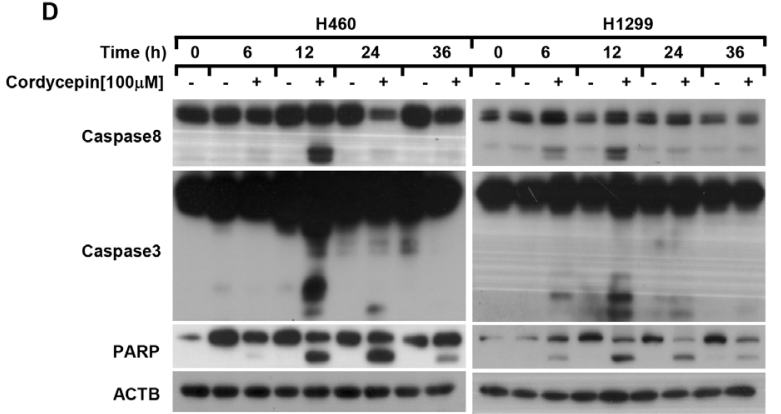

Figure 1: Cordycepin triggers caspase-dependent apoptosis in NSCLC. A. Cell viability of H1792, A549, H1299, H157 and H460 cells was measured by SRB assay after treated with the indicated concentrations of cordycepin for $24 \mathrm{~h}$ and $48 \mathrm{~h}$. B. Flow cytometry analysis was performed in H460 and H1299 cell lines after treated with the indicated concentrations of cordycepin for $24 \mathrm{~h}$. The indicated cells were treated with different concentrations of cordycepin for $12 \mathrm{~h} \mathrm{C}$. or exposed to $100 \mu \mathrm{M}$ cordycepin for the indicated time D., thereafter whole cell lysates were collected for Western blot analysis. Data were normalized to control and represented mean \pm SEM for three independent experiments $\left({ }^{*} \mathrm{p}<0.05,{ }^{* *} \mathrm{p}<0.01,{ }^{* * *} \mathrm{p}<0.001\right)$.

A
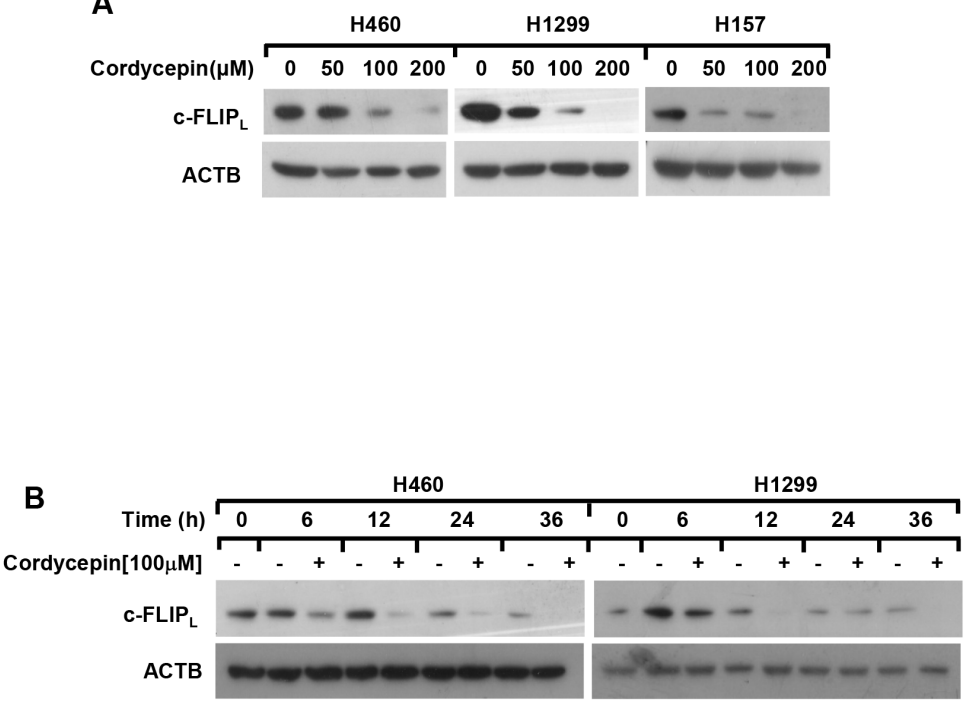
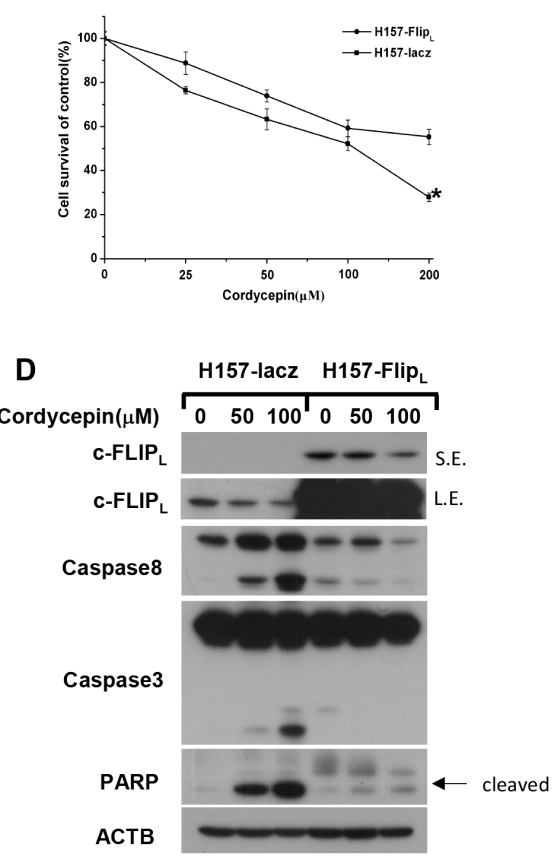

Figure 2: Cordycepin induces apoptosis through down-regulation of $\mathbf{c}-$ FLIP $_{\mathbf{L}}$. The indicated cell lines were disposed with 0 , 50,100 and $200 \mu \mathrm{M}$ cordycepin for $12 \mathrm{~h} \mathrm{~A}$. or treated with $100 \mu \mathrm{M}$ cordycepin for the indicated time B., then the whole-cell protein lysates were harvested and prepared for western blot analysis. C. The viability of H157-FLIP ${ }_{\mathrm{L}}$ and H157-lacz cells were measured by SRB assay after exposed to the indicated concentrations of cordycepin for $24 \mathrm{~h}$. D. H157-FLIP $\mathrm{L}_{\mathrm{L}}$ and H157-lacz cells were treated with $0,50,100 \mu \mathrm{M}$ condycepin for $12 \mathrm{~h}$, then whole cell lysates were collected for western blot analysis. Data represented mean \pm SEM for three independent experiments each. L.E.: long exposure; S.E.: short exposure. ${ }^{*} \mathrm{p}<0.05,{ }^{* *} \mathrm{p}<0.01,{ }^{* * *} \mathrm{p}<0.001$. 


\section{Cordycepin triggers autophagy by inhibiting mTOR pathway}

Because autophagy plays vital role in determining cell fate [12], we wondered whether cordycepin induced autophagy in NSCLC cells. The conversion of LC3B-I to LC3B-II by conjugating with phosphatidylethanolamine is the typical recognition of autophagy. Thus, we detected the expression of LC3B-II formation after treatment with the indicated concentration of cordycepin for $12 \mathrm{~h}$ and found that the conversion of LC3B-II was obviously enhanced in a dose-dependent manner (Figure 3A). Moreover, p62, an autophagy receptor which can bind to specific cargo for degradation in the lysosome during the process of autophagy, was down-regulated in a dose-dependent manner after cordycepin treatment, providing evidence for autophagy occurrence (Figure 3A).

To further supervise the autophagic flux induced by cordycepin, H1299 and H460 cell lines were transfected with Atg5 siRNA to inhibit Atg5 expression and the transformation of LC3B-II was dramatically decreased after Atg5 silence (Figure 3B). Furthermore, when H1299 cells were co-incubated with cordycepin and LY294002, which obstructs the early stage of autophagy, the level of LC3B-II was greatly down-regulated (Figure 3C). On the contrary, co-treatment with cordycepin and chloroquine (CQ), which blocks the late stage of autophagy, enhanced the transformation of LC3B-II (Figure 3C). In conclusion, we deduce that cordycepin induces autophagic flux in human NSCLC cells.

mTOR signaling works as a crucial modulator of autophagy initiation. p-P70S6K and p-4EBP1 exist as the two vital substrates of mTOR [17]. Furthermore, we inspected whether cordycepin-triggered autophagy through mTOR suppression. Human NSCLC cell lines H460, H1299 and H157 were treated with the indicated dosage of cordycepin for $12 \mathrm{~h}$, and the dose-dependent western blot results showed that cordycepin explosion notably declined the levels of p-P70S6K and p-4EBP1 (Figure 3D). Therefore, we speculated that cordycepin induced autophagy via mTOR inhibition.

\section{Cordycepin causes pro-apoptotic autophagy in NSCLC cells}

Since cordycepin induces apoptosis and autophagy in human NSCLC cells, we further explore the relationship between cordycepin-triggered apoptosis and autophagy. Atg 5 siRNA was employed to inhibit Atg 5 expression in NSCLC cells, and then western blot analysis was used to detect apoptosis-related proteins. The data demonstrated that Atg5 silence significantly abrogated cordycepincaused cleavage of caspase- 8 , caspase- 3 and PARP (Figure $4 \mathrm{~A})$. Furthermore, flow cytometry analysis was applied to investigate the effect of autophagy on cordycepininduced apoptosis. Consistently, we found that inhibiting autophagy effectively blocked cordycepin-induced apoptosis in human lung cancer cells (Figure 4B).

To further confirm the interaction between apoptosis and autophagy by cordycepin, H460 and H1299 were pretreated with LY294002, an early inhibitor of autophagy for $1 \mathrm{~h}$, and then exposed to $200 \mu \mathrm{M}$ cordycepin for $12 \mathrm{~h}$. According to Figure 4C, co-treatment with LY294002 and cordycepin apparently decreased the cleavage of caspase 3 and PARP, which was in accordance with the results of flow cytometry analysis (Figure 4D). Consequently, we come to the conclusion that cordycepin-induced autophagy facilitates apoptosis.
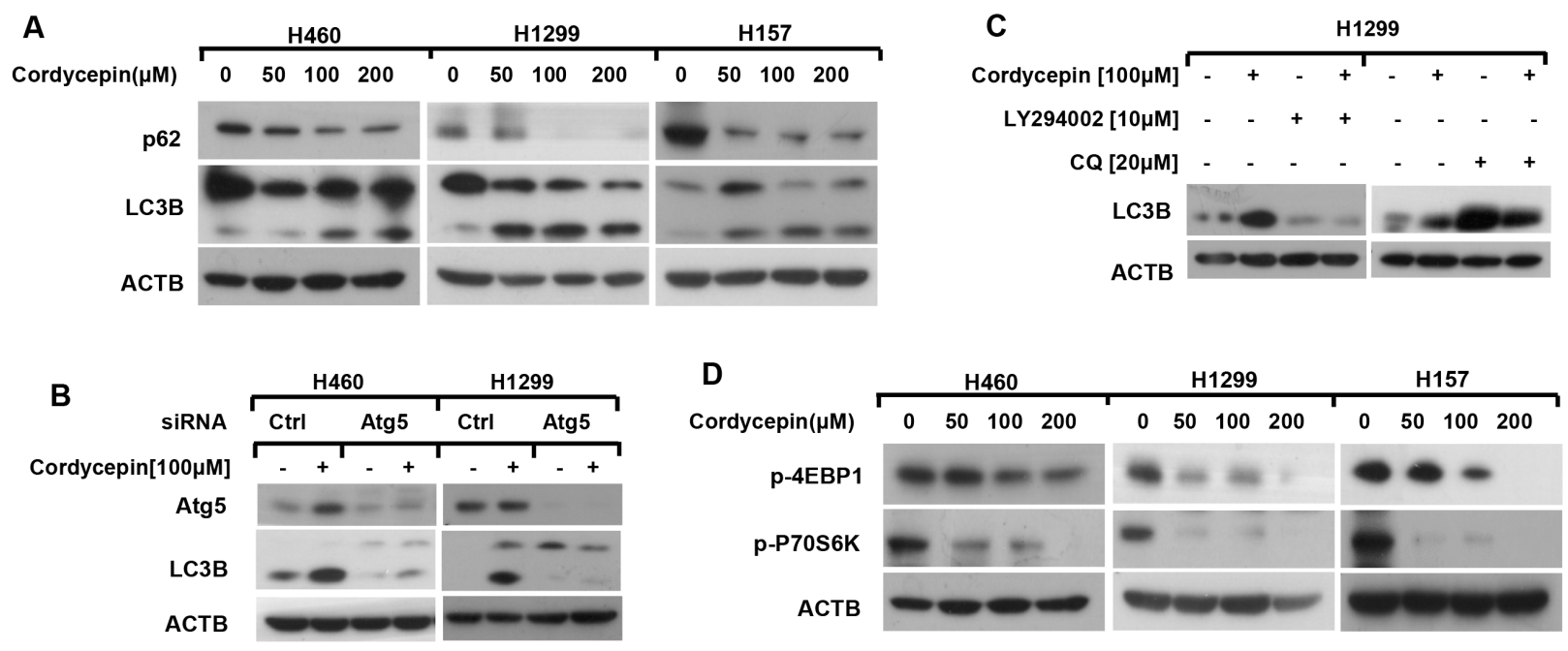

Figure 3: Cordycepin inactivates mTOR and leads to autophagy in NSCLC cells. A and D. H460, H1299 and H157 cell lines were treated with 0, 50, 100 and $200 \mu \mathrm{M}$ cordycepin for $12 \mathrm{~h}$. B. H460 and H1299 cells were conducted with Atg5 siRNA and treated with $100 \mu \mathrm{M}$ cordycepin for $12 \mathrm{~h}$. C. H1299 cells were pretreated with10 $\mu \mathrm{M} \mathrm{LY} 294002$ or $20 \mu \mathrm{M}$ CQ for $1 \mathrm{~h}$ and then exposed to $100 \mu \mathrm{M}$ cordycepin for another $12 \mathrm{~h}$. Then whole-cell protein lysates were harvested and prepared for western blot analysis. 


\section{Cordycepin-triggered autophagy promotes c-FLIP degradation}

As cordycepin induced apoptosis through c-FLIP ${ }_{L}$ down-regulation and stimulated pro-apoptotic autophagy in human NSCLC cells, we speculated that cordycepin influenced $\mathrm{c}-\mathrm{FLIP}_{\mathrm{L}}$ degradation by autophagy, which eventually induced apoptosis. As shown in Figure 5A, Atg5 siRNA was utilized to suppress autophagy, and western blot assay revealed that Atg5 knockdown obviously weakened the decrease of $\mathrm{c}^{-\mathrm{FLIP}_{\mathrm{L}}}$ after cordycepin exposure. Moreover, autophagy inhibitors such as LY294002 or CQ, blocked the upstream or downstream steps of autophagy respectively, were used to examine the impact of autophagy on c-FLIP ${ }_{L}$ degradation. The results showed that either blocking the upstream of autophagy by LY294002 or interdicting the downstream of autophagy by $\mathrm{CQ}$, the decrease of c-FLIP triggered by cordycepin was significantly attenuated (Figure 5B). Hence, we propose that cordycepin treatment triggers autophagy-mediated degradation of c-FLIP , leading to apoptosis eventually in human lung cancer cells (Figure 6).

\section{DISCUSSION}

Recently, many efforts have been done to investigate cordycepin-induced cell death. However, the elaborate molecular mechanism of apoptosis triggered by cordycepin is still poorly deciphered in NSCLC. Thus, the main purposes of our research focused on illuminating the underlying mechanisms of cordycepin-induced apoptosis as well as exploring the relationship between apoptosis and autophagy caused by cordycepin.

It has been reported that cordycepin induces apoptosis through enhancing the expression of JNK, p38 kinase and Bcl-2 pro-apoptotic members [18], increasing the mitochondrial translocation of Bax and the release of cytochrome $\mathrm{C}$, and causing the reactive oxygen species (ROS)-mediated activation of caspases [19]. In our present study, we found that cell viability of human NSCLC cells greatly dropped in both a dose- and a time-dependent fashion after cordycepin incubation, indicating that cordycepin exerted killing effects on human lung cancer cells. In addition, flow cytometery analysis revealed that approximately 40 percentages of $\mathrm{H} 1299$ and H460 cells
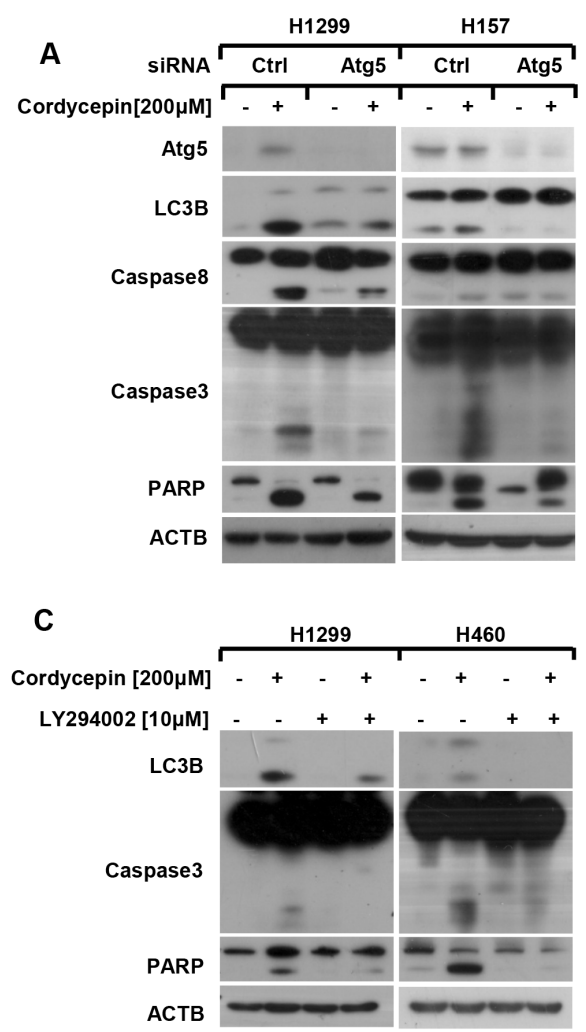

B
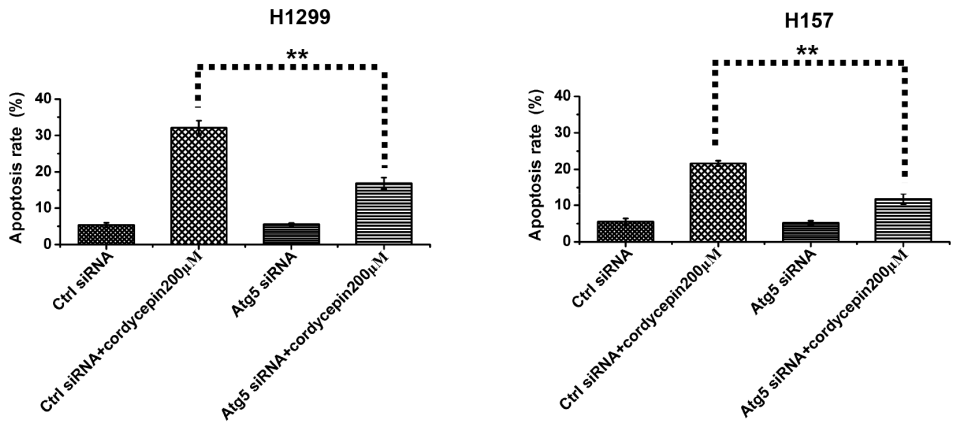

D
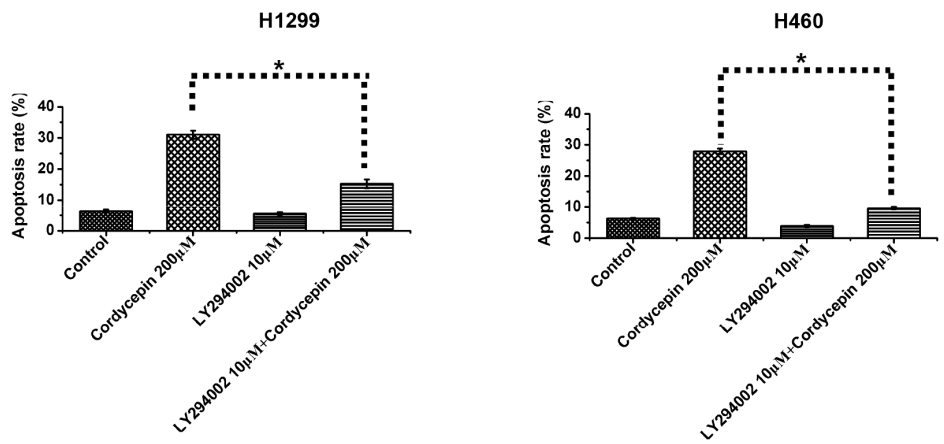

Figure 4: Cordycepin gives rise to pro-apoptotic autophagy in NSCLC cells. H1299 and H157 cell lines were seeded in six-well plates and conducted with Atg5 siRNA transfection on the next day. After Forty-eight hours transfection, cells were exposed to $200 \mu \mathrm{M}$ cordycepin for $12 \mathrm{~h}$. Subsequently, cells were harvested and prepared for western blot analysis A. or subjected to flow cytometry analysis after $24 \mathrm{~h}$ treatment B. H1299 and H460 cell lines were seeded in six-well plates and pretreated with $10 \mu \mathrm{M} \mathrm{LY} 294002$ for $1 \mathrm{~h}$ on the next day, then cells were exposed to $200 \mu \mathrm{M}$ cordycepin for $12 \mathrm{~h}$. Subsequently, cells were harvested and prepared for western blot analysis $\mathbf{C}$. or subjected to flow cytometry analysis after $24 \mathrm{~h}$ treatment $\mathbf{D}$. Data represented mean \pm SEM from three independent experiments $\left({ }^{*} \mathrm{p}<0.05,{ }^{* *} \mathrm{p}<0.01, * * \mathrm{p}<0.001\right)$. 
step into early and late phase apoptosis after treated with $200 \mu \mathrm{M}$ cordycepin for $24 \mathrm{~h}$. And the cleaved forms of caspase-8 (the initiator caspase of extrinsic apoptosis), caspase-9 (the initiator caspase of intrinsic apoptosis), caspase-3 and PARP were also raised in both a dose- and a time-dependent manner. The data suggest that cordycepin induced extrinsic and intrinsic apoptosis in human lung cancer cells.
Furthermore, we inspected the mechanism of cordycepin-induced apoptosis and discovered that the expression of c-FLIP ${ }_{\mathrm{L}}$ was dramatically down-regulated, and the ectopic expression of c-FLIP $_{\mathrm{L}}$ decreased the cleavage of caspase- 8 , caspase- 3 and PARP, protecting H157-FLIP $_{L}$ cells from cordycepin-triggered extrinsic apoptosis. Our results provide detailed clues to explain cordycepin-induced apoptosis.
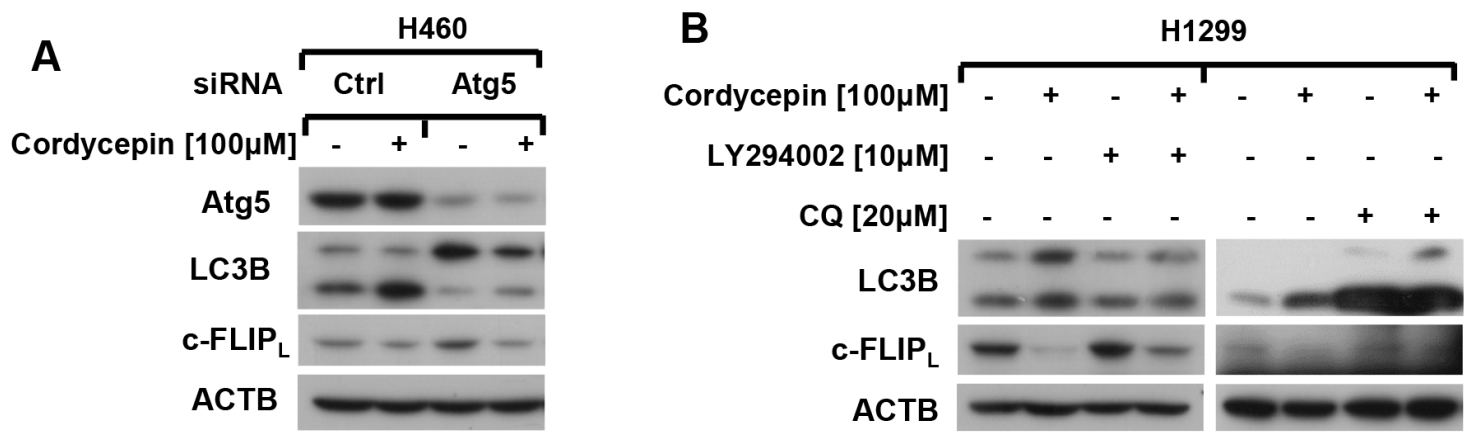

Figure 5: Cordycepin promotes c-FLIP degradation through activating autophagy. A. H460 cells were seeded in six-well plates and treated with Atg5 siRNA on the next day. After Forty-eight hours transfection, cells were exposed to $100 \mu \mathrm{M}$ cordycepin for 12 h. Thereafter, cells were harvested and prepared for western blot analysis. B. H1299 cells were pretreated with $10 \mu \mathrm{M}$ LY294002 or 20 $\mu \mathrm{M}$ CQ for $1 \mathrm{~h}$, and then exposed to $100 \mu \mathrm{M}$ cordycepin for another $12 \mathrm{~h}$. The whole-cell protein lysates were harvested and subjected to western blot analysis.

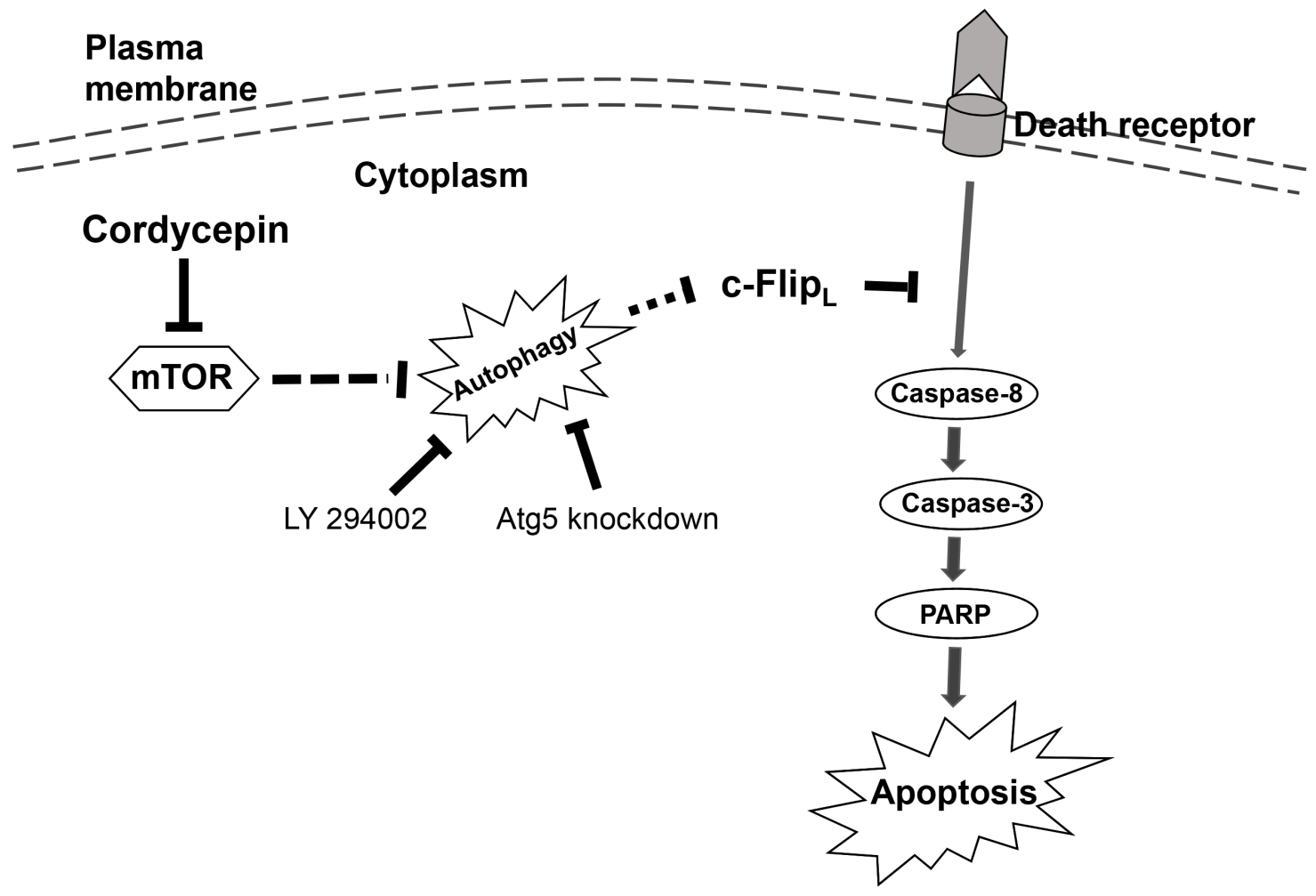

Figure 6: The schematic model of cordycepin-triggered apoptosis and autophagy in human NSCLC cells. Cordycepin treatment stimulates extrinsic apoptosis pathway by inhibiting the expression of c-FLIP ${ }_{\mathrm{L}}$. Furthermore, cordycepin also triggered autophagy through suppression of mTOR signaling pathway. Cordycepin-induced autophagy promoted apoptosis through degrading c-FLIP ${ }_{\mathrm{L}}$. 
Autophagy is a conserved degradation physiological process served to protect cells from the potential damages induced by dysfunctional organelles and protein aggregations [20]. Here, we showed that cordycepin strengthened the transformation of LC3B-II and heightened the degradation of p62, which implied that cordycepin may induce autophagy in lung cancer. Furthermore, to monitor the autophagic flux stimulated by cordycepin, H1299 cells were co-treated with cordycepin and the autophagy inhibitors such as LY294002 and CQ, which blocked the early and the late stages of autophagy respectively. Western blot analysis revealed that the conversion of LC3B-II was greatly decreased after LY294002 co-treatment, while an obvious increase of LC3B-II transformation was occurred after CQ co-incubation. Collectively, these results suggest that cordycepin induces autophagic flux in human lung cancer cells.

mTOR is one of the classical pathways that regulates autophagic induction by two main downstream elements: p70S6K and 4EBP1 [21]. Consistently, western blot assay revealed that the level of $\mathrm{p}-\mathrm{P} 70 \mathrm{~S} 6 \mathrm{~K}$ and $\mathrm{p}-4 \mathrm{EBP} 1$ were down-regulated following cordycepin therapy, indicating that mTOR might participate in cordycepin-induced autophagy. mTOR not only negatively regulate autophagy, but also play an important role in regulating cell survival and apoptosis. Thus, we conjectured that cordycepin-caused mTOR suppressing might account for the induction of early autophagy and subsequent apoptosis in NSCLC cells.

As autophagy and apoptosis are two important cellular processes with complex and intersecting protein networks, extensive investigations have focused on the relationship of them [13]. In some circumstance, autophagy develops a stress adaptation by suppressing apoptosis to avoid cell death [22]. Meanwhile it has been reported that inhibition of autophagy promotes apoptosis and decreases cell viability in LNCaP cells after treated with cordycepin [23]. Recently, Munoz-Pinedo et al. proposed a new term-"Autosis", which is used to define the autophagic cell-death pathway [24]. In our research, we found that inhibiting autophagy by silencing Atg5 expression or LY294002 treatment weakened cordycepininduced apoptosis, indicating cordycepin triggered proapoptotic autophagy in NSCLC cells. However, the molecular mechanism of autophagy exerted pro-apoptotic function in cordycepin-induced cell death is still unclear.

It has been reported that drug-induced autophagy down-regulates c-FLIP and enhances apoptosis in NSCLC cells $[25,26]$. Here, we found that cordycepin induced c-FLIP ${ }_{\mathrm{L}}$-mediated apoptosis and triggered proapoptotic autophagy in human lung cancer cells. Thus, we further investigated the impact of cordycepin-caused autophagy on the degradation of $\mathrm{c}-\mathrm{FLIP}_{\mathrm{L}}$. Western blot analysis showed that the degradation of $c-F_{L} P_{L}$ was dramatically attenuated when autophagy was blocked by Atg5 knockdown. Moreover, the decrease of c-FLIP caused by cordycepin was also greatly weakened after autophagy inhibitors (LY294002 and CQ) treatment. Hence, we propose that cordycepin-caused autophagy promotes c-FLIP ${ }_{\mathrm{L}}$ down-regulation.

In summary, our results demonstrate that cordycepin triggers autophagy-mediated c-FLIP ${ }_{\mathrm{L}}$ degradation and promotes apoptosis in human NSCLC cells. Thus, we illuminate a novel molecular mechanism of apoptosis caused by cordycepin, which may provide theoretical basis for exploitation and application of cordycepin derivatives in cancer treatment.

\section{MATERIALS AND METHODS}

\section{Reagents and antibodies}

Cordycepin ( $>98 \%$ pure) was purchased from Mellon (Dalian China). The stock solution of cordycepin $(0.5 \mathrm{~mol} / \mathrm{L})$ was prepared in DMSO and diluted to the desired concentration with fresh medium immediately before use. PARP, Caspase-8, Caspase-9, pp70S6K, p-4EBP1 and Atg5 antibodies were purchased from Cell Signaling Technology (Danvers, MA). Mcl-1 and Bcl-2 antibodies were purchased from Santa cruz (Santa Cruz, CA). Caspase-3 antibody was purchased from Imgenex (San Diego, CA). $\beta$-actin and LC3B antibodies were purchased from Sigma (Santa Clara, CA). p62 antibody was purchased from Becton, Dickinson and Company (New Jersey, USA).

\section{Cell lines and cell culture}

Human NSCLC cells lines H1792, H1299, H460, $\mathrm{H} 157$ and A549 were initially obtained from the American Type Culture Collection (Manassas, VA). Monolayer cultures of all the cell lines were cultivated in RPMI 1640 supplemented with 5\% FBS in an atmosphere of 95\% air and $5 \% \mathrm{CO}_{2}$ at $37^{\circ} \mathrm{C}$.

\section{Cell survival assay}

Sulforhodamine B (SRB) assay was used to assess cell survival as described earlier [27]. IC50 (half maximal inhibitory concentration) value was determined by the software SPSS.

\section{Western blot analysis}

The preparation of whole-cell protein lysates and the Western blot analysis were carried out as previously described [28].

\section{Establishment of stable cell lines that overexpress c-FLIP $_{\mathrm{L}}$}

c-FLIP ${ }_{L}$ coding regions were amplified by PCR and cloned into the pLenti6 vector (Invitrogen) according to 
the manufacturer's protocol. 293FT cells were used to prepare lentivirus and the selection of H157 cell clones was according to the procedure previously described [29]. For simplicity, the clone that over-expressed c-FLIP $\mathrm{L}_{\mathrm{L}}$ is referred to as $\mathrm{H}_{157-\mathrm{FLIP}_{\mathrm{L}}}$ and the control clone is named as H157-LacZ.

\section{Small interference RNA transfection}

H1299, H157 and H460 cells were seeded in 6-well plates and treated at the confluency of 50\% with a control nonspecific siRNA and Atg5 siRNA using Polyplus transfection reagent according to the manufacturer's recommendations. After $24 \mathrm{~h}$ incubation, cells were treated with $200 \mu \mathrm{M}$ cordycepin and the gene silencing effects were evaluated by western blot analysis. Atg5 siRNA duplexes target the sequences 5'-CCTTTGGCCTAAGAAGAAA-3'.

\section{Flow cytometric analysis}

Cell apoptosis was examined by Annexin V-FITC Apoptosis Detection Kit purchased from Biobox Biotech (Nanjing, China) according to the manufacture's protocol.

\section{Statistical analysis}

Each value is the mean of at least three separate experiments. Student's $t$-test was performed to compare the differences between treated groups and their controls. Difference was considered significant at $\mathrm{P}<0.05$.

\section{Abbreviations}

c-FLIP, cellular FLICE (FADD-like IL-1 $\beta$ converting enzyme)-inhibitory protein; $\mathrm{CQ}$, Chloroquine; DISC, death-inducing signaling complex; mTOR, Mammalian Target of Rapamycin; NSCLC, non-small cell lung cancer; EIF4EBP1, eukaryotic initiation factor 4E binding protein 1; p70S6K, p70 ribosomal protein S6kinase; PARP, poly ADP-ribose polymerase.

\section{ACKNOWLEDGMENTS}

This work was supported by the grants from the National Natural Science Foundation of China (31371402, 81472686 and 31171332) and the Science and technology development plan of Shandong Province (2014GSF118067 and 2016GSF201153).

\section{CONFLICTS OF INTEREST}

The authors declare no conflicts of interest.

\section{REFERENCES}

1. Zhang JX, Han YP, Bai C, Li Q. Notch1/3 and p53/p21 are a potential therapeutic target for APS-induced apoptosis in non-small cell lung carcinoma cell lines. International Journal of clinical and experimental medicine. 2015; 8:12539-12547.

2. Ahn YJ, Park SJ, Lee SG, Shin SC, Choi DH. Cordycepin: selective growth inhibitor derived from liquid culture of Cordyceps militaris against Clostridium spp. Journal of Agricultural and Food Chemistry. 2000; 48:2744-2748.

3. Lee SY, Debnath T, Kim SK, Lim BO. Anti-cancer effect and apoptosis induction of cordycepin through DR3 pathway in the human colonic cancer cell HT-29. Food and Chemical Toxicology. 2013; 60:439-447.

4. Wang XA, Xiang SS, Li HF, Wu, XS, Li ML, Shu YJ, Zhang F, Cao Y, Ye YY, Bao RF, Weng H, Wu WG, Mu JS, Hu YP, Jiang L, Tan ZJ, Lu W, Wang P, Liu YB. Cordycepin induces $\mathrm{S}$ phase arrest and apoptosis in human gallbladder cancer cells. Molecules. 2014; 19:11350-11365.

5. Liao Y, Ling J, Zhang G, Liu F, Tao S, Han Z, Chen S, Chen $\mathrm{Z}$, Le H. Cordycepin induces cell cycle arrest and apoptosis by inducing DNA damage and up-regulation of p53 in Leukemia cells. Cell Cycle. 2015. 14:761-771.

6. Aramwit P, Porasuphatana S, Srichana T, Nakpheng T. Toxicity evaluation of cordycepin and its delivery system for sustained in vitro anti-lung cancer activity. Nanoscale Research Letters. 2015. 10:152.

7. Lowe SW, Lin AW. Apoptosis in cancer. Carcinogenesis. 2000. 21:485-495.

8. Safa AR. Roles of c-FLIP in Apoptosis, Necroptosis, and Autophagy. Journal of carcinogenesis \& mutagenesis. 2013; Suppl 6.

9. Rahman MA, Sultan MT, Islam MR. Apoptosis and cancer: insights molecular mechanisms and treatments. International Journal of Biomolecules \& Biomedicine. 2012; 2:1-16.

10. Mojsa B, Lassot I, Desagher S. Mcl-1 ubiquitination: unique regulation of an essential survival protein. Cells. 2014; 3:418-437.

11. Kroemer G, Marino G, Levine B. Autophagy and the integrated stress response. Molecular Cell. 2010; 40:280-293.

12. Oral O, Akkoc Y, Bayraktar O, Gozuacik D. Physiological and pathological significance of the molecular crosstalk between autophagy and apoptosis. Histology and Histopathology. 2016; 31:497-498.

13. Mukhopadhyay S, Panda PK, Sinha N, Das DN, Bhutia SK. Autophagy and apoptosis: where do they meet? Apoptosis. 2014; 19:555-566.

14. Lauring J, Park BH, Wolff AC. The phosphoinositide-3kinase-Akt-mTOR pathway as a therapeutic target in breast 
cancer. Journal of the National Comprehensive Cancer Network. 2013; 11:670-678.

15. Yang Z, Klionsky DJ. Mammalian autophagy: core molecular machinery and signaling regulation. Current Opinion in Cell Biology. 2010; 22:124-131.

16. Goldar S, Khaniani MS, Derakhshan SM, Baradran B. Molecular mechanisms of apoptosis and roles in cancer development and treatment. Asian Pacific Journal of Cancer Prevention. 2015; 16:2129-2144.

17. Gu L, Xie L, Zuo C, Ma Z, Zhang Y, Zhu Y, Gao J. Targeting mTOR/p70S6K/glycolysis signaling pathway restores glucocorticoid sensitivity to 4E-BP1 null Burkitt Lymphoma. BMC Cancer. 2015; 15:529.

18. He W, Zhang MF, Ye J, Jiang TT, Fang X, Song Y. Cordycepin induces apoptosis by enhancing JNK and p38 kinase activity and increasing the protein expression of Bcl-2 pro-apoptotic molecules. J Zhejiang Univ Sci B. 2010; 11:654-660.

19. Jeong JW, Jin CY, Park C, Hong SH, Kim GY, Jeong YK, Lee JD, Yoo YH, Choi YH. Induction of apoptosis by cordycepin via reactive oxygen species generation in human leukemia cells. Toxicology in Vitro. 2011; 25:817-824.

20. MizushimaI N, Komatsu M. Autophagy: renovation of cells and tissues. Cell. 2011; 147:728-741.

21. Jung CH, Ro SH, Cao J, Otto NM, Kim DH. 2010. mTOR regulation of autophagy. FEBS Letters. 2010; 584:1287-1295.

22. Maiuri MC, Zalckvar E, Kimchi A, Kroemer G. Self-eating and self-killing: crosstalk between autophagy and apoptosis. Nature Reviews Molecular Cell Biology. 2007; 8:741-752.

23. Lee HH, Kim SO, Kim GY, Moon SK, Kim WJ, Jeong YK, Yoo YH, Choi YH. Involvement of autophagy in cordycepin-induced apoptosis in human prostate carcinoma LNCaP cells. Environmental Toxicology and Pharmacology. 2014; 38:239-250.

24. Munoz-Pinedo C, Martin SJ. Autosis: a new addition to the cell death Tower of Babel. Cell Death \& Disease. 2014; 5:e1319.

25. Jennings $\mathrm{Xu}$, Xiuling $\mathrm{Xu}$, Shaoqing Shi, Qiong Wang, Bryanna Saxton, Weiyang He, Xin Gou, Jun-Ho Jang, Toru Nyunoya, Xia Wang, Chengguo Xing, Lin Zhang, Yong Lin. Autophagy-Mediated Degradation of IAPs and c-FLIPL Potentiates Apoptosis Induced by Combination of TRAIL and Chal-24. Journal of Cellular Biochemistry. 2016; 117:1136-1144.

26. Uddin MD. Nazim, Ji-Hong Moon, Ju-Hee Lee, You-Jin Lee, Jae-Won Seol, Seong-Kug Eo, John-Hwa Lee, SangYouel Park. Activation of autophagy flux by metformin downregulates cellular FLICE-like inhibitory protein and enhances TRAIL-induced apoptosis. Oncotarget. 2016; 7:23468-23481. doi: 10.18632/oncotarget.8048.

27. Su L, Liu G, Hao X, Zhong N, Zhong D, Liu X, Singhal $\mathrm{S}$. Death receptor 5 and cellular FLICE-inhibitory protein regulate pemetrexed-induced apoptosis in human lung cancer cells. The European Journal of Cancer. 2011; 47:2471-2478.

28. Liu X, Yue P, Zhou Z, Khuri FR, Sun SY. Death receptor regulation and celecoxib-induced apoptosis in human lung cancer cells. Journal of the National Cancer Institute. 2004; 96:1769-1780

29. Liu X, Yue P, Schonthal AH, Khuri FR, Sun SY. Cellular FLICE-inhibitory protein down-regulation contributes to celecoxib-induced apoptosis in human lung cancer cells. Cancer Research. 2006; 66:11115-11119. 